\title{
ELLIPTIC EQUATIONS WITH CRITICAL SOBOLEV EXPONENTS IN DIMENSION 3
}

\author{
Olivier DRUET \\ Université de Cergy-Pontoise, Département de Mathématiques, Site de Saint-Martin, \\ 2 avenue Adolphe Chauvin, 95302 Cergy-Pontoise cedex, France \\ Received 31 January 2001, revised 6 November 2001
}

ABSTRACT. - We study the existence of minimizing solutions for an elliptic equation with critical Sobolev growth on a smooth bounded domain of $\mathbf{R}^{3}$. We answer in particular two questions of Haïm Brezis. Higher dimensions $n \geqslant 4$ are completely understood thanks to previous works by H. Brezis and L. Nirenberg.

(C) 2002 L'Association Publications de l'Institut Henri Poincaré. Published by Elsevier B.V. All rights reserved

RÉSUMÉ. - On étudie l'existence de solutions minimisantes à une EDP elliptique à croissance de Sobolev critique sur des domaines de l'espace euclidien de dimension 3. On résout en particulier une conjecture de $\mathrm{H}$. Brezis sur le sujet. Les questions analogues en dimensions plus grandes étaient parfaitement comprises depuis des travaux de H. Brezis et L. Nirenberg.

(C) 2002 L'Association Publications de l'Institut Henri Poincaré. Published by Elsevier B.V. All rights reserved

Let $\Omega$ be a smooth bounded domain of $\mathbf{R}^{n}, n \geqslant 3$, and let us consider the following problem:

$$
\text { (E) } \begin{cases}\Delta u+a u=u^{2^{*}-1} & \text { in } \Omega, \\ u>0 & \text { in } \Omega, \\ u=0 & \text { on } \partial \Omega,\end{cases}
$$

where $a$ is a smooth function in $\Omega, 2^{*}=2 n /(n-2)$ is critical for the embeddings of $H_{0}^{1}(\Omega)$ into $L^{q}(\Omega)$ and $\Delta$ is the Euclidean Laplacian with the minus sign convention, that is $\Delta u=-\operatorname{div}(\nabla u)$. We look for solutions of problem $(E)$ which are in $H_{0}^{1}(\Omega)$, the completion of $C_{c}^{\infty}(\Omega)$ for the norm

$$
\|u\|_{H_{0}^{1}(\Omega)}^{2}=\int_{\Omega}|\nabla u|^{2} \mathrm{~d} x .
$$

Any such solution of problem $(E)$ is smooth in $\Omega$ by standard elliptic regularity theory. As a first remark, one should note that, if problem $(E)$ possesses a solution, then the operator $\Delta+a$ must be coercive. By definition, $\Delta+a$ is coercive if its first eigenvalue

E-mail address: Olivier.Druet@math.u-cergy.fr (O. Druet). 
with Dirichlet boundary condition is positive. From now on, we consider functions $a$ such that the operator $\Delta+a$ is coercive. A natural approach to find solutions of $(E)$ is to consider the following minimization problem:

$$
J_{a}=\inf _{u \in C_{c}^{\infty}(\Omega), u \neq 0} \frac{\int_{\Omega}\left(|\nabla u|^{2}+a u^{2}\right) \mathrm{d} x}{\left(\int_{\Omega}|u|^{2^{*}} \mathrm{~d} x\right)^{2 / 2^{*}}} .
$$

If $J_{a}$ is achieved by some $u_{a} \in H_{0}^{1}(\Omega)$, up to changing $u_{a}$ into $\left|u_{a}\right|$ and up to normalization, one gets a smooth solution of $(E)$. Such a solution is referred to as a minimizing solution of $(E)$. It is well known that, in any case,

$$
J_{a} \leqslant K_{n}^{-1},
$$

where $K_{n}$ is the best constant in the $H_{1}^{2}\left(\mathbf{R}^{n}\right)$-Sobolev inequality, defined by

$$
K_{n}^{-1}=\inf _{u \in C_{c}^{\infty}\left(\mathbf{R}^{n}\right), u \neq 0} \frac{\int_{\mathbf{R}^{n}}|\nabla u|^{2} \mathrm{~d} x}{\left(\int_{\mathbf{R}^{n}}|u|^{2^{*}} \mathrm{~d} x\right)^{2 / 2^{*}}} .
$$

Its value, independently computed in [2] and [22], is

$$
K_{n}=\frac{4}{n(n-2)} \omega_{n}^{-2 / n},
$$

where $\omega_{n}$ denotes the volume of the standard unit $n$-sphere. When looking for minimizing solutions of $(E)$, it was shown in Brezis and Nirenberg [4] that the situation changes drastically when passing from dimensions $n \geqslant 4$ to dimension 3. In particular, Brezis and Nirenberg proved that, if $n \geqslant 4$, the following properties are equivalent:

(i) $\exists x \in \Omega, a(x)<0$,

(ii) $J_{a}<K_{n}^{-1}$,

(iii) $J_{a}$ is achieved by some smooth positive function $u_{a}$.

In dimension $n=3$, the situation is more tricky and still open. Available results concern the special case $a \equiv \lambda, \lambda$ a constant. More precisely, Brezis and Nirenberg proved in [4] that for any smooth bounded domain $\Omega$ of $\mathbf{R}^{3}$, there exists $\lambda^{*}(\Omega) \in\left(0, \lambda_{1}(\Omega)\right), \lambda_{1}(\Omega)$ being the first Dirichlet eigenvalue of $\Delta$ in $\Omega$, such that

$$
\begin{aligned}
& J_{\lambda}=K_{3}^{-1} \text { when } \lambda \geqslant-\lambda^{*}(\Omega), \\
& J_{\lambda}<K_{3}^{-1} \text { when }-\lambda_{1}(\Omega)<\lambda<-\lambda^{*}(\Omega)
\end{aligned}
$$

and such that $J_{\lambda}$ is not achieved for $\lambda>-\lambda^{*}(\Omega)$. If $\Omega$ is a ball $B$, they also proved that $\lambda^{*}(B)=\frac{1}{4} \lambda_{1}(B)$ and that $J_{-\frac{1}{4} \lambda_{1}(B)}$ is not achieved.

Under the light of these results, H. Brezis asked in [3] the following question:

If $n=3$, is $J_{a}$ achieved if and only if $J_{a}<K_{3}^{-1}$ as it is the case in dimensions $n \geqslant 4$ ? (Question 5 of [3].)

From now on, $\Omega$ is a smooth bounded domain of $\mathbf{R}^{3}$ and $a \in C^{\infty}(\Omega)$ is such that $\Delta+a$ is coercive. We let $G_{a}: \Omega \times \Omega \backslash\{(x, x), x \in \Omega\} \rightarrow \mathbf{R}^{+}$be the Green function of $\Delta+a$ in 
$\Omega$ with Dirichlet boundary condition. We have, in the sense of distributions,

$$
\begin{array}{ll}
\Delta_{y} G_{a}(x, y)+a G_{a}(x, y)=\delta_{x} & \text { in } \Omega, \\
G_{a}(x, y)=0 & \text { for } y \in \partial \Omega, x \in \Omega,
\end{array}
$$

and $G_{a}$ is symmetric with respect to the two variables. We may write

$$
G_{a}(x, y)=\frac{1}{\omega_{2}|x-y|}+g_{a}(x, y),
$$

where $g_{a} \in C^{0}(\Omega \times \Omega)$ verifies for any $x \in \Omega$ :

$$
\begin{array}{ll}
\Delta_{y} g_{a}(x, y)+a G_{a}(x, y)=0 & \text { in } \Omega, \\
g_{a}(x, y)=-\frac{1}{\omega_{2}|x-y|} & \text { on } \partial \Omega .
\end{array}
$$

By test functions computations (see [19]), one gets that

$$
\exists x \in \Omega, \quad g_{a}(x, x)>0 \Rightarrow J_{a}<K_{3}^{-1} .
$$

The condition that $g_{a}(x, x)$ should be positive somewhere in $\Omega$ plays the role the condition that $a(x)$ should be negative somewhere in $\Omega$ played in the case $n \geqslant 4$. Another natural question then, asked by Brezis in [3], is the following:

Is the converse of (1) true? (Question 7 of [3].)

In this paper, we answer by the affirmative both these questions. More precisely, we prove the following:

THEOREM 0.1. - Let $\Omega$ be a bounded domain of $\mathbf{R}^{3}$ and let a $\in C^{\infty}(\Omega) \cap L^{\infty}(\Omega)$ be such that $\Delta+$ a is coercive. The following properties are equivalent:

(i) $\exists x \in \Omega, g_{a}(x, x)>0$,

(ii) $J_{a}<K_{3}^{-1}$,

(iii) $J_{a}$ is achieved by some smooth positive function $u_{a}$.

Note that, by [19], (i) implies (ii) (see above) and that, by standard minimization techniques, (ii) implies (iii). Note also that Theorem 0.1 has already been proved in [3] and [4] in the case $a \equiv \lambda$ and $\Omega$ is a ball, $\lambda$ a constant. Analoguous results were also proved by the author [8] in the Riemannian setting. The main difference between dimension 3 and higher dimensions is that the first problem is a global one whereas the second problem is a local one. If $n=3$, the condition that there exists $x \in \Omega$ such that $g_{a}(x, x)>0$ is indeed a global condition since the Green function of $\Delta+a$ depends on the values of $a$ on all of $\Omega$ and also on the geometry of $\Omega$. There is another difference between the two cases $n=3$ and $n \geqslant 4$. Following Hebey and Vaugon [15], we say that $a \in C^{\infty}(\Omega)$ is a critical function in $\Omega$ if and only if

$$
\begin{aligned}
& J_{a}=K_{n}^{-1}, \\
& J_{\tilde{a}}<K_{n}^{-1}, \quad \text { for any } \tilde{a} \leqslant a, \tilde{a} \not \equiv a .
\end{aligned}
$$


Then, if $n \geqslant 4$, the constant function $a \equiv 0$ is the only critical function in any smooth bounded domain of $\mathbf{R}^{n}$. Indeed, by [4], if $a$ is a critical function in $\Omega, a(x) \geqslant 0$ for any $x \in \Omega$. Since $J_{0}=K_{n}^{-1}$, the only possibility for $a$ to be a critical function is $a \equiv 0$. On the contrary, as a consequence of our proof of Theorem 0.1 (see below), if $n=3$, then there are critical functions of all shape. More precisely, for any smooth bounded domain $\Omega$ of $\mathbf{R}^{3}$ and any $a \in C^{\infty}(\Omega)$, there exists $B(a) \in \mathbf{R}$ such that $a+B(a)$ is a critical function in $\Omega$.

The proof of the theorem is mainly based on a fine blow-up analysis for sequences of solutions of an elliptic PDE in $\mathbf{R}^{3}$. There are many works about this kind of blow-up analysis: among them, $[1,5,12-16,18,20]$ were a great source of inspiration.

\section{Proof of the theorem}

We first note that for any $a, \tilde{a} \in C^{\infty}(\Omega)$, we have

$$
\tilde{a} \geqslant a, \tilde{a} \not \equiv a \Rightarrow g_{a}(x, x)>g_{\tilde{a}}(x, x), \quad \text { for any } x \in \Omega .
$$

This follows from the Green representation formula: indeed, for any $x \in \Omega$,

$$
\begin{aligned}
g_{a}(x, x)-g_{\tilde{a}}(x, x) & =\int_{\Omega}(\tilde{a}-a)(y) G_{a}(x, y) G_{\tilde{a}}(x, y) \mathrm{d} y, \\
& >0,
\end{aligned}
$$

as soon as $\tilde{a} \geqslant a$ with $\tilde{a} \not \equiv a$. We let now $a \in C^{\infty}(\Omega)$ and we define $B(a) \in \mathbf{R}$ by

$$
\begin{array}{ll}
J_{a+B}<K_{3}^{-1}, & \text { for } B<B(a), \\
J_{a+B} \geqslant K_{3}^{-1}, & \text { for } B \geqslant B(a) .
\end{array}
$$

It is clear that $B(a)$ is well defined, since for $B$ large (for instance $B>-\min a$ ), $J_{a+B} \geqslant K_{3}^{-1}$ and for $B$ small enough (for instance $B$ less than the first eigenvalue of $\Delta+a$ on $\Omega), J_{a+B}<K_{3}^{-1}$. Proving the theorem reduces now to the proof that

(1) there exists $x_{0} \in \Omega$ such that $g_{a+B(a)}\left(x_{0}, x_{0}\right)=0$.

(2) $J_{a+B(a)}$ is not achieved.

Indeed, $J_{a+B}$ is clearly not achieved by any smooth positive $u_{a+B}$ for $B>B(a)$, since otherwise, we would have

$$
\begin{aligned}
J_{a+B(a)} & \leqslant \frac{\int_{\Omega}\left(\left|\nabla u_{a+B}\right|^{2}+(a+B(a)) u_{a+B}^{2}\right) \mathrm{d} x}{\left(\int_{\Omega}\left|u_{a+B}\right|{ }^{6} \mathrm{~d} x\right)^{1 / 3}} \\
& \leqslant J_{a+B}+\frac{\int_{\Omega}(B(a)-B) u_{a+B}^{2} \mathrm{~d} x}{\left(\int_{\Omega}\left|u_{a+B}\right|^{6} \mathrm{~d} x\right)^{1 / 3}} \\
& <K_{n}^{-1}
\end{aligned}
$$

which is in contradiction with the definition of $B(a)$. Thus, if we prove that $J_{a+B(a)}$ is not achieved, then properties (ii) and (iii) of the theorem are equivalent. Next, if we 
prove that there exists $x_{0} \in \Omega$ such that $g_{a+B(a)}\left(x_{0}, x_{0}\right)=0$, we have then by (2) that for any $B<B(a), g_{a+B}\left(x_{0}, x_{0}\right)>0$ which proves that property (ii) of the theorem implies property (i). Since the converse is true by (1), this clearly proves the theorem. Up to changing $a$ into $a+B(a)$, we may assume that $B(a)=0$.

Step 1 . We first prove that $J_{a+B(a)}$ is not achieved. Let us assume by contradiction that there exists $u_{a} \in C^{\infty}(\Omega), u_{a}>0$ in $\Omega$, which achieves $J_{a}$. Then, up to normalization, $u_{a}$ verifies

$$
\begin{array}{ll}
\Delta u_{a}+a u_{a}=K_{3}^{-1} u_{a}^{5} & \text { in } \Omega, \\
u_{a}=0 & \text { on } \partial \Omega,
\end{array}
$$

and

$$
\int_{\Omega} u_{a}^{6} \mathrm{~d} x=1 .
$$

For any $\varphi \in C^{\infty}\left(\mathbf{R}^{3}\right)$, since $J_{a}=K_{3}^{-1}$, we have that for any $\varepsilon>0$,

$$
\left(\int_{\Omega} u_{a}^{6}(1+\varepsilon \varphi)^{6} \mathrm{~d} x\right)^{1 / 3} \leqslant K_{3}\left[\int_{\Omega}\left|\nabla\left(u_{a}(1+\varepsilon \varphi)\right)\right|^{2} \mathrm{~d} x+\int_{\Omega} a u_{a}^{2}(1+\varepsilon \varphi)^{2} \mathrm{~d} x\right] .
$$

We clearly get with (4) that

$$
\begin{aligned}
\left(\int_{\Omega} u_{a}^{6}(1+\varepsilon \varphi)^{6} \mathrm{~d} x\right)^{1 / 3}= & 1+2 \varepsilon \int_{\Omega} u_{a}^{6} \varphi \mathrm{d} x+5 \varepsilon^{2} \int_{\Omega} u_{a}^{6} \varphi^{2} \mathrm{~d} x \\
& -4 \varepsilon^{2}\left(\int_{\Omega} u_{a}^{6} \varphi \mathrm{d} x\right)^{2}+\mathrm{o}\left(\varepsilon^{2}\right) .
\end{aligned}
$$

On the other hand, using (3) and (4),

$$
\begin{aligned}
& K_{3}\left[\int_{\Omega}\left|\nabla\left(u_{a}(1+\varepsilon \varphi)\right)\right|^{2} \mathrm{~d} x+\int_{\Omega} a u_{a}^{2}(1+\varepsilon \varphi)^{2} \mathrm{~d} x\right] \\
& =K_{3}\left[\int_{\Omega} u_{a}\left(\Delta u_{a}+a u_{a}\right)(1+\varepsilon \varphi)^{2} \mathrm{~d} x+\varepsilon^{2} \int_{\Omega} u_{a}^{2}|\nabla \varphi|^{2} \mathrm{~d} x\right] \\
& =\int_{\Omega} u_{a}^{6}(1+\varepsilon \varphi)^{2} \mathrm{~d} x+K_{3} \varepsilon^{2} \int_{\Omega} u_{a}^{2}|\nabla \varphi|^{2} \mathrm{~d} x \\
& =1+2 \varepsilon \int_{\Omega} u_{a}^{6} \varphi \mathrm{d} x+\varepsilon^{2} \int_{\Omega} \varphi^{2} u_{a}^{6} \mathrm{~d} x+K_{3} \varepsilon^{2} \int_{\Omega} u_{a}^{2}|\nabla \varphi|^{2} \mathrm{~d} x .
\end{aligned}
$$

Coming back to (5) with these two last relations and letting $\varepsilon$ go to 0 , we get that

$$
4 \int_{\Omega} \varphi^{2} u_{a}^{6} \mathrm{~d} x \leqslant K_{3} \int_{\Omega} u_{a}^{2}|\nabla \varphi|^{2} \mathrm{~d} x+4\left(\int_{\Omega} u_{a}^{6} \varphi \mathrm{d} x\right)^{2}, \quad \text { for any } \varphi \in C^{\infty}\left(\mathbf{R}^{3}\right) .
$$


We claim now that there exists $(y, t) \in \mathbf{R}^{3} \times \mathbf{R}^{+}$such that:

$$
\begin{aligned}
& F(y, t):=\int_{\Omega} u_{a}^{6} \frac{2 t(x-y)}{1+t^{2}|x-y|^{2}} \mathrm{~d} x=0, \quad \text { for any } i=1,2,3, \\
& G(y, t):=\int_{\Omega} u_{a}^{6} \frac{1-t^{2}|x-y|^{2}}{1+t^{2}|x-y|^{2}} \mathrm{~d} x=0 .
\end{aligned}
$$

This result is the equivalent of a result on the standard sphere proved by Chang and Yang [6]. Following [6], the proof of such a claim may go as follows: one considers $H: \mathbf{R}^{3} \times \mathbf{R} \rightarrow \mathbf{R}^{4}$ defined by

$$
H(y, s)=\left(F\left(y, \frac{s+\sqrt{s^{2}+4}}{2}\right)+y, G\left(y, \frac{s+\sqrt{s^{2}+4}}{2}\right)+s\right) .
$$

One proves, thank to an asymptotic expansion of $F$ and $G$ that $|H(y, s)|^{2} \leqslant|y|^{2}+s^{2}$ as soon as $|y|^{2}+s^{2}$ is large enough. This proves in particular that there exists $R>0$ such that $H(B(0, R)) \subset B(0, R)$. Since $H$ is a continuous function, we may apply Brouwer's fixed point theorem: $H$ has at least one fixed point in $B(0, R)$. But a fixed point of $H$ is just a zero of both $F$ and $G$. This proves the above claim.

We now apply (6) to each $\varphi_{i}, i=1,2,3,4$, where

$$
\begin{aligned}
\varphi_{i}(x) & :=\frac{2 t\left(x^{i}-y^{i}\right)}{1+t^{2}|x-y|^{2}}, \quad \text { for any } i=1,2,3, \\
\varphi_{4}(x) & :=\frac{1-t^{2}|x-y|^{2}}{1+t^{2}|x-y|^{2}} .
\end{aligned}
$$

Since $F=0$ and $G=0$ for the good choice of $(y, t)$, we then obtain:

$$
4 \sum_{i=1}^{4} \int_{\Omega} u_{a}^{6} \varphi_{i}^{2} \mathrm{~d} x \leqslant K_{3} \sum_{i=1}^{4} \int_{\Omega}\left|\nabla \varphi_{i}\right|^{2} u_{a}^{2} \mathrm{~d} x .
$$

But

$$
\sum_{i=1}^{4} \varphi_{i}^{2}=1
$$

and

$$
\sum_{i=1}^{4}\left|\nabla \varphi_{i}\right|^{2}=\frac{12 t^{2}}{\left(1+t^{2}|x-y|^{2}\right)^{2}}
$$

Thus we have

$$
4 \int_{\Omega} u_{a}^{6} \mathrm{~d} x \leqslant 12 K_{3} \int_{\Omega} \frac{t^{2}}{\left(1+t^{2}|x-y|^{2}\right)^{2}} u_{a}^{2} \mathrm{~d} x .
$$


By Hölder's inequalities and (4), we obtain

$$
1 \leqslant 3 K_{3}\left(\int_{\Omega} \frac{t^{3}}{\left(1+t^{2}|x-y|^{2}\right)^{3}} d x\right)^{2 / 3} .
$$

One then easily verifies that

$$
\left(\int_{\mathbf{R}^{3}} \frac{t^{3}}{\left(1+t^{2}|x-y|^{2}\right)^{3}} \mathrm{~d} x\right)^{2 / 3}=\left(3 K_{3}\right)^{-1} .
$$

Since $\Omega$ is bounded, we clearly get the desired contradiction and $J_{a}$ can not be achieved. The following steps deal with the proof that there exists $x_{0} \in \Omega$ such that $g_{a}\left(x_{0}, x_{0}\right)=0$.

Step 2. By the definition of $B(a)$, for any $\varepsilon>0$, we have that

$$
J_{a-\varepsilon}<K_{3}^{-1} .
$$

Remember that we have chosen $B(a)=0$ for all the proof of the theorem. This inequality ensures, this is by now standard, the existence of a minimizer for $J_{a-\varepsilon}$. Up to normalization, we thus have for any $\varepsilon>0$ that there exists some smooth positive function $u_{\varepsilon}$ in $\Omega$ verifying:

$$
\begin{array}{ll}
\Delta u_{\varepsilon}+(a-\varepsilon) u_{\varepsilon}=\lambda_{\varepsilon} u_{\varepsilon}^{5} & \text { in } \Omega, \\
u_{\varepsilon}=0 & \text { on } \partial \Omega, \\
\int_{\Omega} u_{\varepsilon}^{6} \mathrm{~d} x=1, &
\end{array}
$$

where we have set $\lambda_{\varepsilon}=J_{a-\varepsilon}$.

The aim now is to study this sequence $\left(u_{\varepsilon}\right)$ as $\varepsilon$ goes to 0 . First of all, $\left(u_{\varepsilon}\right)$ is bounded in $H_{0}^{1}(\Omega)$ so that, after passing to a subsequence, $u_{\varepsilon}$ converges weakly to some $u_{0}$ in $H_{0}^{1}(\Omega)$. We may also assume that, up to a subsequence, $\lim _{\varepsilon \rightarrow 0} \lambda_{\varepsilon}=\lambda_{0}$, with $\lambda_{0} \leqslant K_{3}^{-1}$ by (7). By passing to the limit in (8), one checks that $u_{0}$ verifies

$$
\begin{array}{ll}
\Delta u_{0}+a u_{0}=\lambda_{0} u_{0}^{5} & \text { in } \Omega, \\
u_{0}=0 & \text { on } \partial \Omega .
\end{array}
$$

Moreover, by weak convergence properties,

$$
\int_{\Omega} u_{0}^{6} \mathrm{~d} x \leqslant 1
$$

Since $J_{a}=K_{3}^{-1}$, we have that 


$$
\left(\int_{\Omega} u_{0}^{6} \mathrm{~d} x\right)^{1 / 3} \leqslant K_{3}\left(\int_{\Omega}\left|\nabla u_{0}\right|^{2} \mathrm{~d} x+\int_{\Omega} a u_{0}^{2} \mathrm{~d} x\right)=\lambda_{0} K_{3} \int_{\Omega} u_{0}^{6} \mathrm{~d} x,
$$

where we have used the equation verified by $u_{0}$. If $u_{0} \neq \equiv 0$, we obtain that $\lambda_{0}=K_{3}^{-1}$ and that $\int_{\Omega} u_{0}^{6} \mathrm{~d} x=1$ which exactly means that $J_{a}$ is achieved by $u_{0}$. Since we proved in Step 1 that $J_{a}$ was not achieved,

$$
u_{\varepsilon} \rightarrow 0 \quad \text { in } H_{0}^{1}(\Omega) .
$$

By the compactness of the embedding of $H_{0}^{1}(\Omega)$ into $L^{2}(\Omega)$, we have that

$$
\lim _{\varepsilon \rightarrow 0} \int_{\Omega} u_{\varepsilon}^{2} \mathrm{~d} x=0
$$

We let $x_{\varepsilon}$ be a point of $\Omega$ where $u_{\varepsilon}$ achieves its maximum and we set

$$
u_{\varepsilon}\left(x_{\varepsilon}\right)=\mu_{\varepsilon}^{-1 / 2}=\sup _{\Omega} u_{\varepsilon}
$$

Since

$$
1=\int_{\Omega} u_{\varepsilon}^{6} \mathrm{~d} x \leqslant \frac{\int_{\Omega} u_{\varepsilon}^{2} \mathrm{~d} x}{\mu_{\varepsilon}^{2}}
$$

it is clear by (9) that $\mu_{\varepsilon} \rightarrow 0$ as $\varepsilon \rightarrow 0$. Combining standard results of elliptic theory (namely results of [21] and [10] or [17]), one gets that

$$
\lim _{\varepsilon \rightarrow 0} \mu_{\varepsilon}^{1 / 2} u_{\varepsilon}\left(\mu_{\varepsilon} x+x_{\varepsilon}\right)=\left(1+\frac{\omega_{3}^{-2 / 3}}{4}|x|^{2}\right)^{-1 / 2} \quad \text { in } C_{\mathrm{loc}}^{0}\left(\mathbf{R}^{3}\right) \cap L_{\mathrm{loc}}^{6}\left(\mathbf{R}^{3}\right) .
$$

In particular, we have that

$$
\lim _{\varepsilon \rightarrow 0} \frac{d\left(x_{\varepsilon}, \partial \Omega\right)}{\mu_{\varepsilon}}=+\infty
$$

and that

$$
\lim _{R \rightarrow+\infty} \lim _{\varepsilon \rightarrow 0} \int_{B\left(x_{\varepsilon}, R \mu_{\varepsilon}\right)} u_{\varepsilon}^{6} \mathrm{~d} x=1 .
$$

By bootstrap techniques and since $u_{\varepsilon}$ verifies (8), one also obtains that

$$
\lim _{\varepsilon \rightarrow 0} u_{\varepsilon}=0 \quad \text { in } C_{\text {loc }}^{0}\left(\bar{\Omega} \backslash\left\{x_{0}\right\}\right)
$$

where $x_{0}=\lim _{\varepsilon \rightarrow 0} x_{\varepsilon}$, up to the extraction of a subsequence.

Step 3 (Weak pointwise estimates). We claim that there exists $C>0$ such that for any $\varepsilon>0$,

$$
\left|x-x_{\varepsilon}\right|^{1 / 2} u_{\varepsilon}(x) \leqslant C, \quad \text { for any } x \in \Omega \text {. }
$$


A similar estimate was obtained in [16] and [20]. We follow here the proof of [7]. Let us note, for $x \in \Omega$,

$$
w_{\varepsilon}(x)=\left|x-x_{\varepsilon}\right|^{1 / 2} u_{\varepsilon}(x)
$$

and let $y_{\varepsilon}$ be a point of $\Omega$ where $w_{\varepsilon}$ achieves its maximum. We assume by contradiction that

$$
w_{\varepsilon}\left(y_{\varepsilon}\right)=\sup _{\Omega} w_{\varepsilon} \rightarrow+\infty \quad \text { as } \varepsilon \rightarrow 0
$$

Clearly, by (11),

$$
\lim _{\varepsilon \rightarrow 0} \frac{\left|x_{\varepsilon}-y_{\varepsilon}\right|}{\mu_{\varepsilon}}=+\infty
$$

and, by (16),

$$
\lim _{\varepsilon \rightarrow 0} \frac{\left|x_{\varepsilon}-y_{\varepsilon}\right|}{u_{\varepsilon}\left(y_{\varepsilon}\right)^{-2}}=+\infty
$$

Since $\Omega$ is bounded, we also clearly have that $\lim _{\varepsilon \rightarrow 0} u_{\varepsilon}\left(y_{\varepsilon}\right)=+\infty$ and thanks to (14), that $\lim _{\varepsilon \rightarrow 0}\left|x_{\varepsilon}-y_{\varepsilon}\right|=0$. We set now, for $x \in \tilde{\Omega}_{\varepsilon}=\left\{\frac{y-y_{\varepsilon}}{u_{\varepsilon}\left(y_{\varepsilon}\right)^{-2}}, y \in \Omega\right\}$,

$$
\tilde{u}_{\varepsilon}(x)=u_{\varepsilon}\left(y_{\varepsilon}\right)^{-1} u_{\varepsilon}\left(u_{\varepsilon}\left(y_{\varepsilon}\right)^{-2} x+y_{\varepsilon}\right) \text {. }
$$

It is clear that

$$
\begin{array}{ll}
\Delta \tilde{u}_{\varepsilon}+\left(a\left(u_{\varepsilon}\left(y_{\varepsilon}\right)^{-2} x+y_{\varepsilon}\right)-\varepsilon\right) u_{\varepsilon}\left(y_{\varepsilon}\right)^{-4} \tilde{u}_{\varepsilon}=\lambda_{\varepsilon} \tilde{u}_{\varepsilon}^{5} & \text { in } \tilde{\Omega}_{\varepsilon}, \\
\tilde{u}_{\varepsilon}=0 & \text { on } \partial \tilde{\Omega}_{\varepsilon} .
\end{array}
$$

For any $x \in B(0,1) \cap \tilde{\Omega}_{\varepsilon}$, we have that

$$
\begin{aligned}
\tilde{u}_{\varepsilon}(x) & =u_{\varepsilon}\left(y_{\varepsilon}\right)^{-1} u_{\varepsilon}\left(u_{\varepsilon}\left(y_{\varepsilon}\right)^{-2} x+y_{\varepsilon}\right) \\
& \leqslant \frac{w_{\varepsilon}\left(u_{\varepsilon}\left(y_{\varepsilon}\right)^{-2} x+y_{\varepsilon}\right)}{w_{\varepsilon}\left(y_{\varepsilon}\right)} \frac{\left|x_{\varepsilon}-y_{\varepsilon}\right|^{1 / 2}}{\left|u_{\varepsilon}\left(y_{\varepsilon}\right)^{-2} x+y_{\varepsilon}-x_{\varepsilon}\right|^{1 / 2}},
\end{aligned}
$$

so that

$$
\tilde{u}_{\varepsilon}(x)^{2} \leqslant \frac{\left|x_{\varepsilon}-y_{\varepsilon}\right|}{\left|u_{\varepsilon}\left(y_{\varepsilon}\right)^{-2} x+y_{\varepsilon}-x_{\varepsilon}\right|}
$$

by the definition of $y_{\varepsilon}$. Then, by (18), we obtain that, for $\varepsilon$ small enough,

$$
\tilde{u}_{\varepsilon}(x)^{2} \leqslant 2, \quad \text { for any } x \in B(0,1) \cap \tilde{\Omega}_{\varepsilon} .
$$

We let now $\eta \in C_{c}^{\infty}(B(0,1))$ and $k \geqslant 1$. Some integration by parts, using Eq. (20) and the fact that $\tilde{u}_{\varepsilon}$ is uniformly bounded in $B(0,1) \cap \tilde{\Omega}_{\varepsilon}$, lead to the following:

$$
\int_{B(0,1) \cap \tilde{\Omega}_{\varepsilon}}\left|\nabla\left(\eta \tilde{u}_{\varepsilon}^{k / 2}\right)\right|^{2} \mathrm{~d} x \leqslant C\left(\|\nabla \eta\|_{\infty}^{2}+\|\Delta \eta\|_{\infty}+k\right) \int_{B(0,1) \cap \tilde{\Omega}_{\varepsilon}} \tilde{u}_{\varepsilon}^{k} \mathrm{~d} x .
$$


Using Sobolev's inequality, we obtain that

$$
\left(\int_{B(0,1) \cap \tilde{\Omega}_{\varepsilon}}\left(\eta \tilde{u}_{\varepsilon}^{k / 2}\right)^{6} \mathrm{~d} x\right)^{1 / 3} \leqslant C\left(\|\nabla \eta\|_{\infty}^{2}+\|\Delta \eta\|_{\infty}+k\right) \int_{B(0,1) \cap \tilde{\Omega}_{\varepsilon}} \tilde{u}_{\varepsilon}^{k} \mathrm{~d} x,
$$

where $C$ is some constant independent of $\varepsilon, k$ and $\eta$. For any $i$, we let $k_{i}=6 \times 3^{i}$ and we take some $\eta_{i}$ such that $\eta_{i}=1$ on $B\left(0, \frac{1}{2}+2^{-i}\right), \eta_{i}=0$ on $B\left(0, \frac{1}{2}+2^{-i-1}\right)$, $\left\|\nabla \eta_{i}\right\|_{\infty}^{2}+\left\|\Delta \eta_{i}\right\|_{\infty} \leqslant C 4^{-i-1}$. Applying the above inequality by induction on $i$, we obtain that

$$
\sup _{B\left(0, \frac{1}{2}\right) \cap \tilde{\Omega}_{\varepsilon}} \tilde{u}_{\varepsilon} \leqslant C\left(\int_{B(0,1) \cap \tilde{\Omega}_{\varepsilon}} \tilde{u}_{\varepsilon}^{6} \mathrm{~d} x\right)^{1 / 6},
$$

where $C>0$ is independent of $\varepsilon$. Since $\tilde{u}_{\varepsilon}(0)=1$, we have obtained the existence of some $C>0$ independent of $\varepsilon$ such that

$$
\int_{B\left(0, \frac{1}{2}\right) \cap \tilde{\Omega}_{\varepsilon}} \tilde{u}_{\varepsilon}^{6} \mathrm{~d} x \geqslant C
$$

Then, we write that for $\varepsilon$ small,

$$
1=\int_{\Omega} u_{\varepsilon}^{6} \mathrm{~d} x \geqslant \int_{\Omega \cap B\left(y_{\varepsilon}, u_{\varepsilon}\left(y_{\varepsilon}\right)^{-2}\right)} u_{\varepsilon}^{6} \mathrm{~d} x+\int_{B\left(x_{\varepsilon}, R \mu_{\varepsilon}\right)} u_{\varepsilon}^{6} \mathrm{~d} x,
$$

since $B\left(y_{\varepsilon}, u_{\varepsilon}\left(y_{\varepsilon}\right)^{-2}\right) \cap B\left(x_{\varepsilon}, R \mu_{\varepsilon}\right)=\emptyset$ for $\varepsilon$ small enough: this is the content of (17) and (18). By passing to $\lim _{R \rightarrow+\infty} \lim _{\varepsilon \rightarrow 0}$ in the right hand side, we obtain, with (13) and the estimate above, a contradiction. Thus (15) is proved.

A simple adaptation of the above proof gives that

$$
\lim _{R \rightarrow+\infty} \lim _{\varepsilon \rightarrow 0} \sup _{x \in \Omega \backslash B\left(x_{\varepsilon}, R \mu_{\varepsilon}\right)}\left|x_{\varepsilon}-x\right|^{1 / 2} u_{\varepsilon}(x)=0 .
$$

Step 4 (Ruling out boundary accumulation). In this step, we prove that the accumulation point $x_{0}$ cannot be on the boundary of $\Omega$. Let us assume by contradiction that

$$
v_{\varepsilon}:=d\left(x_{\varepsilon}, \partial \Omega\right) \rightarrow 0 \quad \text { as } \varepsilon \rightarrow 0 .
$$

We set for $x \in \Omega_{\varepsilon}=\left\{\frac{y-x_{\varepsilon}}{v_{\varepsilon}}, y \in \Omega_{\varepsilon}\right\}$,

$$
v_{\varepsilon}(x)=v_{\varepsilon}^{1 / 2} u_{\varepsilon}\left(v_{\varepsilon} x+x_{\varepsilon}\right) .
$$

Then $v_{\varepsilon}$ verifies the following:

$$
\begin{array}{ll}
\Delta v_{\varepsilon}+\left(a_{\varepsilon}-\varepsilon\right) v_{\varepsilon}^{2} v_{\varepsilon}=\lambda_{\varepsilon} v_{\varepsilon}^{5} & \text { in } \Omega_{\varepsilon}, \\
v_{\varepsilon}=0 & \text { on } \partial \Omega_{\varepsilon},
\end{array}
$$


where we have set $a_{\varepsilon}(x)=a\left(v_{\varepsilon} x+x_{\varepsilon}\right)$. Moreover,

$$
\int_{\Omega_{\varepsilon}} v_{\varepsilon}^{6} \mathrm{~d} x=1
$$

One also checks that (11), (13), (15) and (21) are scale invariant and thus continue to hold with $v_{\varepsilon}, \mu_{\varepsilon} / v_{\varepsilon}, \Omega_{\varepsilon}$ and 0 instead of $u_{\varepsilon}, \mu_{\varepsilon}, \Omega$ and $x_{\varepsilon}$ (note that, by (12), $\mu_{\varepsilon} / v_{\varepsilon} \rightarrow 0$ as $\varepsilon \rightarrow 0)$. Since $\Omega$ is smooth and with (22), we have that, up to a rotation,

$$
\left.\lim _{\varepsilon \rightarrow 0} \Omega_{\varepsilon}=\Omega_{0}=\mathbf{R}^{2} \times\right]-\infty ; 1[
$$

For any $R>0$, we let

$$
\eta_{\varepsilon}(R)=\sup _{x \in \overline{\Omega_{\varepsilon}} \cap \overline{B(0, R) \backslash B(0, R / 2)}} v_{\varepsilon} .
$$

We let also $L_{\varepsilon}$ be the operator

$$
L_{\varepsilon} u=\Delta u+\left(a_{\varepsilon}-\varepsilon\right) v_{\varepsilon}^{2} u-\lambda_{\varepsilon} v_{\varepsilon}^{4} u
$$

and we compute $L_{\varepsilon}\left(|x|^{\nu-1}\right)$ for some $0<v<1$ on $B(0, R) \cap \Omega_{\varepsilon} \backslash B\left(0, \tilde{R} \frac{\mu_{\varepsilon}}{v_{\varepsilon}}\right)$ where $R$ is fixed and $\tilde{R}$ will be fixed later on. Easy computations lead to

$$
L_{\varepsilon}\left(|x|^{\nu-1}\right)=|x|^{\nu-3}\left(v(1-v)+\left(a_{\varepsilon}-\varepsilon\right) v_{\varepsilon}^{2}|x|^{2}-\lambda_{\varepsilon}|x|^{2} v_{\varepsilon}^{4}\right) .
$$

Thanks to (21), we may choose $\tilde{R}$ such that for $\varepsilon$ small enough,

$$
\lambda_{\varepsilon}|x|^{2} v_{\varepsilon}^{4} \leqslant \frac{1}{2} v(1-v) \quad \text { on } B(0, R) \cap \Omega_{\varepsilon} \backslash B\left(0, \tilde{R} \frac{\mu_{\varepsilon}}{v_{\varepsilon}}\right) .
$$

With (11), we may find $C(R, v)$ such that for any $\varepsilon>0$ and any $x$ on the boundary of $B(0, R) \cap \Omega_{\varepsilon} \backslash B\left(0, \tilde{R} \frac{\mu_{\varepsilon}}{v_{\varepsilon}}\right)$,

$$
v_{\varepsilon}(x) \leqslant C(R, v)\left(\left(\frac{\mu_{\varepsilon}}{v_{\varepsilon}}\right)^{1 / 2-v}+\eta_{\varepsilon}(R)\right)|x|^{\nu-1} .
$$

If we let $G_{\varepsilon}(x)$ be the right-hand side of the above equation, we have obtained that

$$
\begin{array}{ll}
L_{\varepsilon}\left(G_{\varepsilon}-v_{\varepsilon}\right) \geqslant 0 & \text { in } B(0, R) \cap \Omega_{\varepsilon} \backslash B\left(0, \tilde{R} \frac{\mu_{\varepsilon}}{v_{\varepsilon}}\right), \\
G_{\varepsilon}-v_{\varepsilon} \geqslant 0 & \text { on } \partial\left(B(0, R) \cap \Omega_{\varepsilon} \backslash B\left(0, \tilde{R} \frac{\mu_{\varepsilon}}{v_{\varepsilon}}\right)\right),
\end{array}
$$

since $L_{\varepsilon} v_{\varepsilon}=0$. This leads to

$$
L_{\varepsilon}\left(G_{\varepsilon}-v_{\varepsilon}\right)^{-} \times\left(G_{\varepsilon}-v_{\varepsilon}\right)^{-} \leqslant 0,
$$


where $\left(G_{\varepsilon}-v_{\varepsilon}\right)^{-}$denotes the negative part of $\left(G_{\varepsilon}-v_{\varepsilon}\right)$. Integrating by parts, we get that

$$
\begin{aligned}
& \int\left|\nabla\left(G_{\varepsilon}-v_{\varepsilon}\right)^{-}\right|^{2} \mathrm{~d} x+v_{\varepsilon}^{2} \int\left(a_{\varepsilon}-\varepsilon\right)\left[\left(G_{\varepsilon}-v_{\varepsilon}\right)^{-}\right]^{2} \mathrm{~d} x \\
& \quad \leqslant \lambda_{\varepsilon} \int v_{\varepsilon}^{4}\left[\left(G_{\varepsilon}-v_{\varepsilon}\right)^{-}\right]^{2} \mathrm{~d} x \\
& \quad<\lambda_{\varepsilon}\left(\int\left[\left(G_{\varepsilon}-v_{\varepsilon}\right)^{-}\right]^{6} \mathrm{~d} x\right)^{1 / 3},
\end{aligned}
$$

where we used (24) and where the integrals are taken over $B(0, R) \cap \Omega_{\varepsilon} \backslash B\left(0, \tilde{R} \frac{\mu_{\varepsilon}}{v_{\varepsilon}}\right)$. This clearly violates the definition of $\lambda_{\varepsilon}$ unless $\left(G_{\varepsilon}-v_{\varepsilon}\right)^{-}=0$. Thus we have that

$$
v_{\varepsilon}(x) \leqslant C(R, v)\left(\left(\frac{\mu_{\varepsilon}}{v_{\varepsilon}}\right)^{1 / 2-v}+\eta_{\varepsilon}(R)\right)|x|^{\nu-1}
$$

for any $x \in B(0, R) \cap \Omega_{\varepsilon}$. Indeed, by (11), this inequality obviously holds on $B\left(0, \tilde{R} \frac{\mu_{\varepsilon}}{v_{\varepsilon}}\right)$.

Let us now prove that

$$
\eta_{\varepsilon}(R) \ll\left(\frac{\mu_{\varepsilon}}{v_{\varepsilon}}\right)^{1 / 2-v} .
$$

Assume that, on the contrary, there exists $C>0$ such that

$$
\eta_{\varepsilon}(R) \geqslant C\left(\frac{\mu_{\varepsilon}}{v_{\varepsilon}}\right)^{1 / 2-v},
$$

so that we may rewrite (26) as

$$
v_{\varepsilon}(x) \leqslant C(r, v) \eta_{\varepsilon}(R)|x|^{\nu-1} .
$$

Then, for any compact subset $K$ of $\overline{B(0, R)} \cap \overline{\Omega_{\varepsilon}} \backslash\{0\}$, there exists $C(K)$ such that

$$
\left(\frac{v_{\varepsilon}(x)}{\eta_{\varepsilon}(R)}\right) \leqslant C(K), \quad \text { for any } x \in K .
$$

By Harnack's inequality, we clearly get the existence of some $D(K)>0$ (for any compact subset $K$ of $\Omega_{\varepsilon} \backslash\{0\}$ ) such that

$$
\left(\frac{v_{\varepsilon}(x)}{\eta_{\varepsilon}(1 / 2)}\right) \leqslant D(K), \quad \text { for any } x \in K .
$$

By standard elliptic theory, see Theorems 8.24 and 8.29 of [11], we obtain then that $\left(v_{\varepsilon} / \eta_{\varepsilon}(1 / 2)\right)$ is bounded in $C_{\text {loc }}^{0, \eta}\left(\overline{\Omega_{\varepsilon}} \backslash\{0\}\right)$. Thus, by Ascoli's theorem, after passing to a subsequence,

$$
\lim _{\varepsilon \rightarrow 0}\left(\frac{v_{\varepsilon}}{\eta_{\varepsilon}(1 / 2)}\right)=G_{0} \quad \text { in } C_{\mathrm{loc}}^{0}\left(\overline{\Omega_{0}} \backslash\{0\}\right),
$$


where we have extended $v_{\varepsilon}$ by 0 outside $\Omega_{\varepsilon}$. Moreover, $G_{0} \not \equiv 0$ and $G_{0}$ verifies:

$$
\begin{array}{ll}
\Delta G_{0}=0 & \text { in } \Omega_{0} \backslash\{0\}, \\
G_{0}=0 & \text { on } \partial \Omega_{0} .
\end{array}
$$

Since $G_{0} \not \equiv 0$, by the maximum principle, $G_{0}$ must clearly be singular. Moreover, by (29), the only possible singularity is at 0 . Thus

$$
G_{0}(x)=\frac{\lambda}{|x|}+b(x)
$$

for some positive constant $\lambda$ and some smooth harmonic function $b$ in $\Omega_{0}$ with $b=$ $-\lambda /|x|$ on $\partial \Omega_{0}$. Integrating (23) on $B(0, \delta)$ for $\delta>0$ small enough, we obtain that

$$
-\int_{\partial B(0, \delta)} \partial_{\nu} v_{\varepsilon} \mathrm{d} \sigma+v_{\varepsilon}^{2} \int_{B(0, \delta)}\left(a_{\varepsilon}-\varepsilon\right) v_{\varepsilon} \mathrm{d} x=\lambda_{\varepsilon} \int_{B(0, \delta)} v_{\varepsilon}^{5} \mathrm{~d} x,
$$

where $v$ denotes the outer normal to $B(0, \delta)$. By (29) and (30), using Lebesgue's dominated convergence theorem, we have that

$$
\eta_{\varepsilon}\left(\frac{1}{2}\right)^{-1} \lambda_{\varepsilon} \int_{B(0, \delta)} v_{\varepsilon}^{5} \mathrm{~d} x \rightarrow-\int_{\partial B(0, \delta)} \partial_{\nu} G_{0} \mathrm{~d} \sigma .
$$

For $\delta$ small enough, $-\int_{\partial B(0, \delta)} \partial_{\nu} G_{0} \mathrm{~d} \sigma$ is positive. Thus

$$
\eta_{\varepsilon}\left(\frac{1}{2}\right) \leqslant C \int_{B(0, \delta)} v_{\varepsilon}^{5} \mathrm{~d} x \leqslant C\left(\frac{\mu_{\varepsilon}}{v_{\varepsilon}}\right)^{1 / 2}+C \int_{B(0, \delta) \backslash B\left(0, R \frac{\mu_{\varepsilon}}{v_{\varepsilon}}\right)} v_{\varepsilon}^{5} \mathrm{~d} x,
$$

for any $R>0$ provided $\varepsilon$ is small enough: we have here used (11). Next, by (29),

$$
\begin{aligned}
\int_{B(0, \delta) \backslash B\left(0, R \frac{\mu_{\varepsilon}}{v_{\varepsilon}}\right)} v_{\varepsilon}^{5} \mathrm{~d} x & \leqslant C \eta_{\varepsilon}\left(\frac{1}{2}\right) \int_{B(0, \delta) \backslash B\left(0, R \frac{\mu_{\varepsilon}}{v_{\varepsilon}}\right)}|x|^{\nu-1} v_{\varepsilon}^{4} \mathrm{~d} x \\
& \leqslant C \eta_{\varepsilon}\left(\frac{1}{2}\right)\left(\int_{\Omega_{\varepsilon} \backslash B\left(0, R \frac{\mu_{\varepsilon}}{v_{\varepsilon}}\right)} v_{\varepsilon}^{6} \mathrm{~d} x\right)^{2 / 3}\left(\int_{B(0, \delta)}|x|^{3 v-3} \mathrm{~d} x\right)^{1 / 3} \\
& \leqslant C \eta_{\varepsilon}\left(\frac{1}{2}\right)\left(\int_{\Omega_{\varepsilon} \backslash B\left(0, R \frac{\mu_{\varepsilon}}{v_{\varepsilon}}\right)} v_{\varepsilon}^{6} \mathrm{~d} x\right)^{2 / 3} .
\end{aligned}
$$

Coming back to the next to last estimate on $\eta_{\varepsilon}(1 / 2)$ and choosing $R$ large enough so that $\int_{\Omega_{\varepsilon} \backslash B\left(0, R \frac{\mu_{\varepsilon}}{v_{\varepsilon}}\right)} v_{\varepsilon}^{6} \mathrm{~d} x$ is small (this is possible by (13)), we obtain the existence of $C>0$ such that

$$
\eta_{\varepsilon}(R) \leqslant C\left(\frac{\mu_{\varepsilon}}{v_{\varepsilon}}\right)^{1 / 2}
$$


which is clearly in contradiction with (28) since $\mu_{\varepsilon} / \nu_{\varepsilon}$ goes to 0 as $\varepsilon$ goes to 0 . Thus we have proved (27).

We want now to prove that (26) still holds for $v=0$. Of course, with the same argument than above, we will then clearly have that

$$
C^{-1} \eta_{\varepsilon}\left(\frac{1}{2}\right) \leqslant\left(\frac{\mu_{\varepsilon}}{\nu_{\varepsilon}}\right)^{1 / 2} \leqslant C \eta_{\varepsilon}\left(\frac{1}{2}\right)
$$

for some $C>1$ independent of $\varepsilon$. We let $\left(z_{\varepsilon}\right)$ be a sequence of points of $\overline{B(0, R)} \cap \overline{\Omega_{\varepsilon}}$. The aim now is to prove that there exists $C(R)>0$ such that

$$
\left|z_{\varepsilon}\right| v_{\varepsilon}\left(z_{\varepsilon}\right)\left(\left(\frac{\mu_{\varepsilon}}{v_{\varepsilon}}\right)^{1 / 2}+\eta_{\varepsilon}(R)\right)^{-1} \leqslant C(R) .
$$

We distinguish three cases.

Case 1: $\left|z_{\varepsilon}\right| v_{\varepsilon} / \mu_{\varepsilon} \rightarrow \delta$ as $\varepsilon \rightarrow 0$. By (11), we clearly have (31) in this case.

Case 2: $\left|z_{\varepsilon}\right| \rightarrow R^{\prime}$ as $\varepsilon \rightarrow 0$ with $R / 2<R^{\prime} \leqslant R$. By the definition of $\eta_{\varepsilon}(R)$, we clearly have

$$
\left|z_{\varepsilon}\right| v_{\varepsilon}\left(z_{\varepsilon}\right) \eta_{\varepsilon}(R)^{-1} \leqslant R
$$

so that (31) is valid in this case.

Case 3: $\left|z_{\varepsilon}\right| \rightarrow R^{\prime}$ as $\varepsilon \rightarrow 0$ with $R^{\prime} \leqslant R / 2$ and $\left|z_{\varepsilon}\right| v_{\varepsilon} / \mu_{\varepsilon} \rightarrow+\infty$ as $\varepsilon \rightarrow 0$. We let $H_{\varepsilon}$ be the Green function of $\Delta-\varepsilon_{0}$ with Dirichlet boundary condition on $\Omega_{\varepsilon} \cap B(0, R)$ for some $\varepsilon_{0}>0$ small enough. By Green's representation formula, we may write that

$$
v_{\varepsilon}\left(z_{\varepsilon}\right)=\int_{\Omega_{\varepsilon} \cap B(0, R)} H_{\varepsilon}\left(z_{\varepsilon}, y\right)\left(\Delta v_{\varepsilon}(y)-\varepsilon_{0} v_{\varepsilon}(y)\right) \mathrm{d} y-\int_{\Omega_{\varepsilon} \cap \partial B(0, R)} \partial_{\nu} H_{\varepsilon}\left(z_{\varepsilon}, y\right) v_{\varepsilon}(y) \mathrm{d} \sigma_{y},
$$

where $v$ denotes the outer normal of $\partial B(0, R)$. By standard elliptic theory, since $\Omega_{\varepsilon} \rightarrow \Omega_{0}$ as $\varepsilon \rightarrow 0$ and since $\left|z_{\varepsilon}\right| \leqslant 2 R / 3$, we get that

$$
-\int_{\Omega_{\varepsilon} \cap \partial B(0, R)} \partial_{\nu} H_{\varepsilon}\left(z_{\varepsilon}, y\right) v_{\varepsilon}(y) \mathrm{d} \sigma_{y} \leqslant C(R) \eta_{\varepsilon}(R) .
$$

On the other hand, using (23), we may write that

$$
\begin{aligned}
& \int_{\Omega_{\varepsilon} \cap B(0, R)} H_{\varepsilon}\left(z_{\varepsilon}, y\right)\left(\Delta v_{\varepsilon}(y)-\varepsilon_{0} v_{\varepsilon}(y)\right) \mathrm{d} y \\
& \quad=\int_{\Omega_{\varepsilon} \cap B(0, R)} H_{\varepsilon}\left(z_{\varepsilon}, y\right)\left[\lambda_{\varepsilon} v_{\varepsilon}^{5}-\left(a_{\varepsilon} v_{\varepsilon}^{2}-\varepsilon v_{\varepsilon}^{2}+\varepsilon_{0}\right) v_{\varepsilon}\right] \mathrm{d} y .
\end{aligned}
$$

Since, by the maximum principle, $H_{\varepsilon}\left(z_{\varepsilon}, y\right) \leqslant 1 /\left(\omega_{2}\left|z_{\varepsilon}-y\right|\right)$, we get that, for $\varepsilon$ small enough,

$$
v_{\varepsilon}\left(z_{\varepsilon}\right) \leqslant C \int_{\Omega_{\varepsilon} \cap B(0, R)}\left|z_{\varepsilon}-y\right|^{-1} v_{\varepsilon}^{5} \mathrm{~d} y+C(R) \eta_{\varepsilon}(R) .
$$


But, by (26) and (27),

$$
\begin{aligned}
\int_{\Omega_{\varepsilon} \cap B(0, R)}\left|z_{\varepsilon}-y\right|^{-1} v_{\varepsilon}^{5} \mathrm{~d} y & \leqslant \int_{B\left(z_{\varepsilon},\left|z_{\varepsilon}\right| / 2\right)}\left|z_{\varepsilon}-y\right|^{-1} v_{\varepsilon}^{5} \mathrm{~d} y+2\left|z_{\varepsilon}\right|^{-1} \int_{\Omega_{\varepsilon} \cap B(0, R)} v_{\varepsilon}^{5} \mathrm{~d} y \\
& \leqslant C(R, v)^{5}\left(\frac{\mu_{\varepsilon}}{v_{\varepsilon}}\right)^{5 / 2-5 v}\left|z_{\varepsilon}\right|^{5 v-3}+C\left|z_{\varepsilon}\right|^{-1}\left(\frac{\mu_{\varepsilon}}{v_{\varepsilon}}\right)^{1 / 2},
\end{aligned}
$$

where we have also used (11). This leads to

$$
\left|z_{\varepsilon}\right| v_{\varepsilon}\left(z_{\varepsilon}\right)\left(\left(\frac{\mu_{\varepsilon}}{v_{\varepsilon}}\right)^{1 / 2}+\eta_{\varepsilon}(R)\right)^{-1} \leqslant C(R)+C\left(\frac{\mu_{\varepsilon}}{v_{\varepsilon}}\right)^{2-5 v}\left|z_{\varepsilon}\right|^{5 v-2} .
$$

Since $\left|z_{\varepsilon}\right| v_{\varepsilon} / \mu_{\varepsilon} \rightarrow+\infty$ as $\varepsilon \rightarrow 0$, taking $v<2 / 5$, we have obtained (31) in this third case.

Thus, (31) is proved. As already mentionned, this is equivalent to

$$
v_{\varepsilon}(x) \leqslant C(R)\left(\frac{\mu_{\varepsilon}}{v_{\varepsilon}}\right)^{1 / 2}|x|^{-1}
$$

for any $x \in \Omega_{\varepsilon} \cap B(0, R) \backslash\{0\}$. By Theorems 8.24 and 8.29 of [11], we then get that

$$
\left(\frac{\mu_{\varepsilon}}{v_{\varepsilon}}\right)^{-1 / 2} v_{\varepsilon} \rightarrow H_{0} \quad \text { in } C_{\mathrm{loc}}^{0}\left(\overline{\Omega_{0}} \backslash\{0\}\right),
$$

where $H_{0}$ is a nonzero harmonic function in $\Omega_{0} \backslash\{0\}$ which vanishes on the boundary of $\Omega_{0}$. This enforces $H_{0}$ to be singular at the origin. Then

$$
H_{0}=\frac{\lambda}{|x|}+b(x)
$$

where $\lambda$ is some positive constant and $b$ is a smooth harmonic function on $\Omega_{0}$ which satisfies $b=-\lambda /|x|$ on $\partial \Omega_{0}$. By the maximum principle, $b$ is everywhere negative and, in particular,

$$
b(0)<0 .
$$

We apply now the Pohozãev identity to $v_{\varepsilon}$ on $B(0,1 / 2)$. This leads to

$$
\begin{aligned}
& v_{\varepsilon}^{2} \int_{B(0,1 / 2)}\left(a_{\varepsilon}-\varepsilon\right) v_{\varepsilon}^{2} \mathrm{~d} x+\frac{3}{2} v_{\varepsilon}^{2} \int_{B(0,1 / 2)}\left(x^{k} \partial_{k} a_{\varepsilon}\right) v_{\varepsilon}^{2} \mathrm{~d} x \\
& =\frac{1}{4} v_{\varepsilon}^{2} \int_{\partial B(0,1 / 2)}\left(a_{\varepsilon}-\varepsilon\right) v_{\varepsilon}^{2} \mathrm{~d} \sigma-\frac{\lambda_{\varepsilon}}{12} \int_{\partial B(0,1 / 2)} v_{\varepsilon}^{6} \mathrm{~d} \sigma \\
& \quad+\frac{1}{2} \int_{\partial B(0,1 / 2)}\left(\frac{1}{2}\left|\nabla v_{\varepsilon}\right|^{2}-\left(\partial_{\nu} v_{\varepsilon}\right)^{2}\right) \mathrm{d} \sigma-\frac{1}{2} \int_{\partial B(0,1 / 2)} v_{\varepsilon} \partial_{\nu} v_{\varepsilon} \mathrm{d} \sigma .
\end{aligned}
$$


Multiplying both sides by $\left(\mu_{\varepsilon} / \nu_{\varepsilon}\right)^{-1}$ and passing to the limit as $\varepsilon \rightarrow 0$ by using (32) and (33), we get that

$$
\int_{\partial B(0,1 / 2)}\left(\frac{1}{2}\left|\nabla H_{0}\right|^{2}-\left(\partial_{\nu} H_{0}\right)^{2}\right) \mathrm{d} \sigma-\int_{\partial B(0,1 / 2)} H_{0} \partial_{\nu} H_{0} \mathrm{~d} \sigma=0 .
$$

Since $\Delta b=0$, it is easy to check that the left-hand side of this relation is just $\lambda \omega_{2} b(0)$. Thus $b(0)=0$ : this is in contradiction with (34). Thus $x_{0} \notin \partial \Omega$.

Step 5. We let now $\varepsilon_{0}>0$ be such that the operator $\Delta+\left(a-\varepsilon_{0}\right)$ is coercive on $\Omega$ and we denote by $G_{a-\varepsilon_{0}}$ its Green's function with Dirichlet boundary condition on $\Omega$. We let also $L_{\varepsilon}$ be the operator defined by $L_{\varepsilon} u=\Delta u+a u-\lambda_{\varepsilon} u_{\varepsilon}^{4} u$. We compute $L_{\varepsilon} G_{a-\varepsilon_{0}}\left(x_{\varepsilon}, x\right)^{1-v}$ on $\Omega \backslash\left\{x_{\varepsilon}\right\}$ for some $0<v<1$. We easily obtain that

$$
\frac{L_{\varepsilon} G_{a-\varepsilon_{0}}\left(x_{\varepsilon}, x\right)^{1-v}}{G_{a-\varepsilon_{0}}\left(x_{\varepsilon}, x\right)^{1-v}}=\varepsilon_{0}-\varepsilon-\lambda_{\varepsilon} u_{\varepsilon}^{4}+v(1-v) \frac{\left|\nabla G_{a-\varepsilon_{0}}\left(x_{\varepsilon}, x\right)\right|^{2}}{G_{a-\varepsilon_{0}}\left(x_{\varepsilon}, x\right)^{2}} .
$$

Since $x_{0} \notin \partial \Omega$, it is easy to see that there exists $C>0, \rho>0$ such that

$$
\left|x-x_{\varepsilon}\right| \leqslant \rho \Rightarrow \frac{\left|\nabla G_{a-\varepsilon_{0}}\left(x_{\varepsilon}, x\right)\right|^{2}}{G_{a-\varepsilon_{0}}\left(x_{\varepsilon}, x\right)^{2}} \geqslant C\left|x-x_{\varepsilon}\right|^{-2} \text {. }
$$

Then we have

$$
\frac{L_{\varepsilon} G_{a-\varepsilon_{0}}\left(x_{\varepsilon}, x\right)^{1-v}}{G_{a-\varepsilon_{0}}\left(x_{\varepsilon}, x\right)^{1-v}} \geqslant \begin{cases}C v(1-v)\left|x_{\varepsilon}-x\right|^{-2}-\lambda_{\varepsilon} u_{\varepsilon}^{4} & \text { in } B\left(x_{\varepsilon}, \rho\right), \\ \left(\varepsilon_{0}-\varepsilon\right)-\lambda_{\varepsilon} u_{\varepsilon}^{4} & \text { in } \Omega \backslash B\left(x_{\varepsilon}, \rho\right) .\end{cases}
$$

Using (14) and (21), we get the existence of some $R(v)>0$ such that for $\varepsilon$ small enough, $L_{\varepsilon} G_{a-\varepsilon_{0}}\left(x_{\varepsilon}, x\right)^{1-v}>0$ on $\Omega \backslash B\left(x_{\varepsilon}, R(v) \mu_{\varepsilon}\right)$. By standard properties of the Green function, since $x_{0} \notin \partial \Omega$, there exists $C>1$ such that $C^{-1} \leqslant\left|x_{\varepsilon}-x\right| \times$ $G_{a-\varepsilon_{0}}\left(x_{\varepsilon}, x\right) \leqslant C$, for any $x \in \Omega$. Then, by (11), there exists $C(v)>0$ such that $u_{\varepsilon}(x) \leqslant C(v) \mu_{\varepsilon}^{1 / 2-v} G_{a-\varepsilon_{0}}\left(x_{\varepsilon}, x\right)^{1-v}$, for any $x \in \partial B\left(0, R(v) \mu_{\varepsilon}\right)$. We deduce from the three last relations, as it was done in the previous step, the following:

$$
u_{\varepsilon}(x) \leqslant C(v) \mu_{\varepsilon}^{1 / 2-v}\left|x_{\varepsilon}-x\right|^{\nu-1}, \quad \text { for any } x \in \Omega \backslash\left\{x_{\varepsilon}\right\} .
$$

Now, by the Green representation formula, for any sequence $\left(z_{\varepsilon}\right)$ of points of $\Omega$, we may write that

$$
u_{\varepsilon}\left(z_{\varepsilon}\right)=\int_{\Omega} G_{a-\varepsilon_{0}}\left(z_{\varepsilon}, y\right)\left[\Delta u_{\varepsilon}(y)+\left(a(y)-\varepsilon_{0}\right) u_{\varepsilon}(y)\right] \mathrm{d} y .
$$

Similar computations to those developed in Step 4 lead to the fundamental estimate:

$$
\left|x-x_{\varepsilon}\right| \mu_{\varepsilon}^{-1 / 2} u_{\varepsilon}(x) \leqslant C .
$$


A similar fundamental estimate was obtained in $[12,14,16]$. The proof presented above follows [9]. We deduce then by standard elliptic theory that

$$
\mu_{\varepsilon}^{-1 / 2} u_{\varepsilon} \rightarrow \lambda G_{a}\left(x_{0}, .\right) \quad \text { in } C_{\mathrm{loc}}^{2}\left(\Omega \backslash\left\{x_{0}\right\}\right),
$$

where $\lambda$ is some positive real number. Applying Pohozãev's identity to $u_{\varepsilon}$ in $B\left(x_{\varepsilon}, \delta\right)$, $\delta>0$ small, one gets that

$$
\begin{aligned}
& \int_{B\left(x_{\varepsilon}, \delta\right)}\left[(a-\varepsilon)+\frac{3}{2}\left(x-x_{\varepsilon}\right)^{k} \partial_{k} a\right] u_{\varepsilon}^{2} \mathrm{~d} x \\
& =\frac{\delta}{2} \int_{\partial B\left(x_{\varepsilon}, \delta\right)}(a-\varepsilon) u_{\varepsilon}^{2} \mathrm{~d} \sigma-\frac{\lambda_{\varepsilon}}{6} \delta \int_{\partial B\left(x_{\varepsilon}, \delta\right)} u_{\varepsilon}^{6} \mathrm{~d} \sigma \\
& \quad+\delta \int_{\partial B\left(x_{\varepsilon}, \delta\right)}\left(\frac{1}{2}\left|\nabla u_{\varepsilon}\right|^{2}-\left(\partial_{\nu} u_{\varepsilon}\right)^{2}\right) \mathrm{d} \sigma-\frac{1}{2} \int_{\partial B\left(x_{\varepsilon}, \delta\right)} u_{\varepsilon} \partial_{\nu} u_{\varepsilon} \mathrm{d} \sigma .
\end{aligned}
$$

Multiplying both sides by $\mu_{\varepsilon}^{-1}$ and passing to the limit as $\varepsilon \rightarrow 0$ by using (35) and (36), we obtain that for any $\delta>0$,

$$
\begin{aligned}
& \int_{B\left(x_{0}, \delta\right)}\left(a+\frac{3}{2}\left(x-x_{0}\right)^{k} \partial_{k} a\right) G_{a}\left(x_{0}, x\right)^{2} \mathrm{~d} x \\
& =\frac{\delta}{2} \int_{\partial B\left(x_{0}, \delta\right)} a G_{a}\left(x_{0}, x\right)^{2} \mathrm{~d} \sigma-\frac{1}{2} \int_{\partial B\left(x_{0}, \delta\right)} G_{a}\left(x_{0}, x\right) \partial_{\nu} G_{a}\left(x_{0}, x\right) \mathrm{d} \sigma \\
& \quad+\delta \int_{\partial B\left(x_{0}, \delta\right)}\left(\frac{1}{2}\left|\nabla G_{a}\left(x_{0}, x\right)\right|^{2}-\left(\partial_{\nu} G_{a}\left(x_{0}, x\right)\right)^{2}\right) \mathrm{d} \sigma .
\end{aligned}
$$

Computing an expansion as $\delta$ goes to 0 to both sides of this relation, one finally gets that $g_{a}\left(x_{0}, x_{0}\right)=0$ which ends the proof of the theorem.

\section{Acknowledgements}

It is my pleasure to express my deep thanks to H. Brezis who put to my attention these questions while I was visiting Rutgers University in the Fall of 1997. I would also like to thank F. Robert and E. Hebey for fruitful discussions we had during the preparation of this work, and for their helpful comments on the manuscript.

\section{REFERENCES}

[1] F.V. Atkinson, L.A. Peletier, Elliptic equations with nearly critical growth, J. Differential Equations 70 (1987) 349-365.

[2] T. Aubin, Problèmes isopérimétriques et espaces de Sobolev, J. Differential Geom. 11 (1976) 573-598.

[3] H. Brézis, Elliptic equations with limiting Sobolev exponents. The impact of topology, Comm. Pure Appl. Math. 39 (1986) 17-39. 
[4] H. Brézis, L. Nirenberg, Positive solutions of nonlinear elliptic equations involving critical Sobolev exponents, Comm. Pure Appl. Math. 36 (1983) 437-477.

[5] H. Brézis, L.A. Peletier, Asymptotics for elliptic equations involving critical Sobolev exponents, in: L. Modica, F. Colombini, A. Marino, S. Spagnolo (Eds.), Partial Differential Equations and the Calculus of Variations, Birkhaüser, Basel, 1989.

[6] S.Y.A. Chang, P.C. Yang, Compactness of isospectral conformal metrics in $S^{3}$, Comment. Math. Helv. 64 (1989) 363-374.

[7] O. Druet, The best constants problem in Sobolev inequalities, Math. Annalen 314 (1999) 327-346.

[8] O. Druet, Optimal Sobolev inequalities and extremal functions, The three-dimensional case, Indiana University Mathematics Journal (to appear).

[9] O. Druet, F. Robert, Asymptotic profile for the sub-extremals of the sharp Sobolev inequality on the sphere, Comm. PDE 26 (5-6) (2001) 743-778.

[10] B. Gidas, W. Ni, L. Nirenberg, Symmetry of positive solutions of nonlinear elliptic equations in $R^{n}$, in: L. Nachbin (Ed.), Mathematical Analysis and Applications, Academic Press, 1981, pp. 370-401.

[11] D. Gilbarg, N. Trüdinger, Elliptic Partial Differential Equations of Second Order, Springer, Berlin, 1977.

[12] Z.C. Han, Asymptotic approach to singular solutions for nonlinear elliptic equations involving critical Sobolev exponent, Ann. I.H.P., Analyse non-linéaire 8 (2) (1991) 159 174.

[13] E. Hebey, Asymptotic behavior of positive solutions of quasilinear elliptic equations with critical Sobolev growth, J. Differential Integral Equations 13 (2000) 1073-1080.

[14] E. Hebey, M. Vaugon, The best constant problem in the Sobolev embedding theorem for complete Riemannian manifolds, Duke Math. J. 79 (1995) 235-279.

[15] E. Hebey, M. Vaugon, From best constants to critical functions, Math. Z. 237 (2001) 737767.

[16] Y.Y. Li, Prescribing scalar curvature on $S^{n}$ and related problems, part I, J. Differential Equations 120 (1995) 319-420.

[17] M. Obata, The conjectures on conformal transformations of Riemannian manifolds, J. Differential Geom. 6 (1971) 247-258.

[18] F. Robert, Asymptotic behaviour of a nonlinear elliptic equation with critical Sobolev exponent: the radial case, Adv. Differential Equations 6 (7) (2001) 821-846.

[19] R. Schoen, Conformal deformation of a Riemannian metric to constant scalar curvature, J. Differential Geom. 20 (1984) 479-495.

[20] R. Schoen, D. Zhang, Prescribed scalar curvature on the $n$-sphere, Calc. Var. PDE 4 (1996) $1-25$.

[21] M. Struwe, A global compactness result for elliptic boundary value problems involving limiting nonlinearities, Math. Z. 187 (1984) 511-517.

[22] G. Talenti, Best constants in Sobolev inequality, Ann. Math. Pura Appl. 110 (1976) 353372. 